\title{
Productivité des caprins dans les systèmes de production agricole du Delta du fleuve Sénégal
}

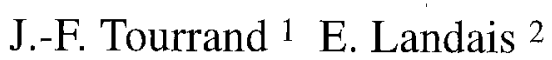

\section{Mots-clés}

Caprin - Chevreau - Productivité Alimentation - Complémentation Elevage - Zootechnie - Sénégal.

\begin{abstract}
Résumé
A travers l'analyse de la productivité, les auteurs prësentent les principaux paramètres zootechniques de cheptel caprin du Delta du fleuve Sénégal, et les comparent aux références disponibles pour la même et quelques autres grandes zones agro-écologiques tropicales. Avec une production annuelle moyenne par chèvre inférieure à un chevreau $(0,85)$ qui pèse à trois mois un peu moins de $8 \mathrm{~kg}$, les performances sont relativement faibles, quoique conformes aux normes régionales. Les auteurs présentent également les pratiques alimentaires qui permettent aux éleveurs peuls du Delta d'améliorer de manière sensible la productivité de leurs troupeaux, d'environ 35 à 40 p. 100, et de valoriser ainsi le potentiel productif de leur cheptel caprin.
\end{abstract}

\section{INTRODUCTION}

\section{Contexte de l'étude : présentation de l'élevage caprin} dans le Delta

Le Delta du fleuve Sénégal ou Waalo est situé dans la zone nordsahélienne de l'Afrique de l'Ouest, en amont de l'embouchure du 'fleuve du même nom, comme l'indique la figure 1. En raison de la présence de vastes bas-fonds exploitables par les bovins en saison sèche, cette région avait jadis une vocation pastorale très marquée. L'élevage des caprins y occupait une place privilégiée, notamment dans la vie agricole des pasteurs peuls et maures (1). Les profondes modifications de l'espace agro-pastoral que cette région a connues aux cours des trente dernières années ont partiellement affecté cet élevage (10). Ces modifications sont essentiellement liécs au dćvcloppement de l'irrigation qui s'est traduit par l'aménagement hydro-agricole des terres de bas-fonds traditionnellement exploitées par le cheptel en saison sèche $(6,10)$.

En 1990, le cheptel caprin du Delta comptait environ 25000 têtes (10), soit un nombre proche de celui estimé en 1965 par Audru (1). La quasi-totalité de ce cheptel est entre les mains des Peuls, les pasteurs maures ayant fui la région lors du conflit armé sénégalo-mauritanien de 1989. Associé à l'élevage des bovins et des ovins, il constitue toujours l'une des principales composantes des systèmes de production agricole propres à cette ethnie (12).

Le mode de conduite des caprins varie peu d'un élevage à l'autre, en dehors du régime alimentaire qui est examiné plus loin. Dans le Delta, chaque exploitation peule ou gallé assure la gestion de son cheptel caprin et dispose d'un enclos pour le parcage de nuit. La taille du cheptel varie selon les exploitations. La majorité des troupeaux compte entre 20 et 80 têtes. Le matin, le troupeau caprin,

1. CIRAD-EMVT, Campus international de Baillarguet, BP 5035, 34032 Montpellier, Cedex 1, France

2. INRA-SAD, Route de Saint-Cyr, 78026 Versailles, Cedex, France

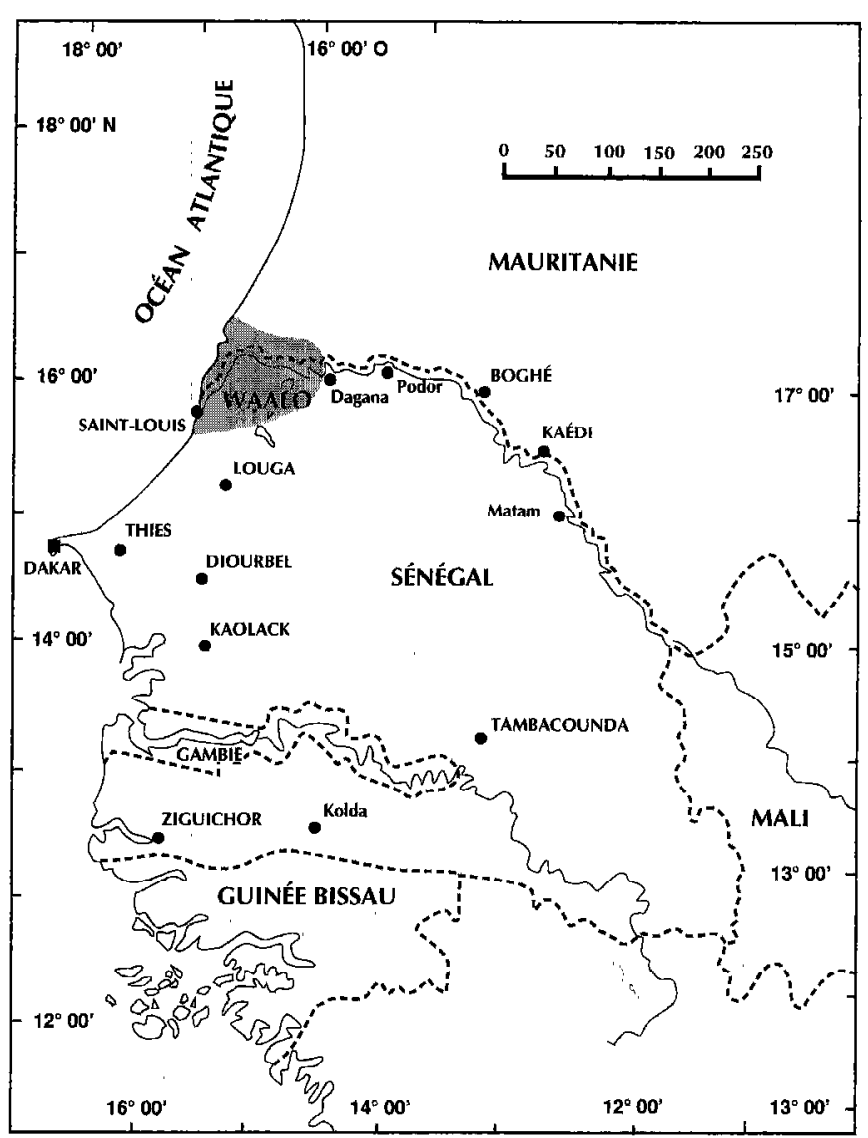

Figure 1 : Delta du fleuve Sénégal ou Waalo

associé au troupeau ovin de l'exploitation, part au pâturage sous la responsabilité d'un berger. Chaque famille organise son calendrier pastoral en fonction des disponibilités fourragères existantes. Les caprins pâturent principalement sur les terres hautes, sableuses, où 
se développe un tapis de graminées annuelles pendant la saison des pluies. En saison sèche, l'apport fourrager des ligneux, dont les gousses et les feuilles sont particulièrement recherchées par les caprins, vient compenser la faible valeur alimentaire des pailles desséchées des graminées. Il faut également mentionner les parcours post-culturaux, principaux lieux de pâture des troupeaux bovins en saison sèche, où l'on rencontre également les quelques troupeaux caprins qui ne disposent pas d'autres parcours à proximité.

En milieu peul, la traite des chèvres est de rigueur lorsque la production laitière du cheptel bovin n'assure pas la couverture des besoins familiaux. Elle a lieu le matin avant le départ au pâturage. En saison sèche, dans quelques troupeaux, exclusivement les chèvres en lactation reçoivent après la traite un complément d'alimentation, composé le plus souvent de farine basse de riz, à raison de 200 à $300 \mathrm{~g}$ par chèvre et par jour durant une période de 30 à 45 jours. Ce complément correspond en moyenne à un apport journalier par animal d'environ 0,22 UFL et $19 \mathrm{~g}$ MAD (CIRADEMVT, 1988, cité par Tourrand (10)). Cette pratique de complémentation a un effet majeur sur la production laitière et sur la productivité du cheptel caprin.

\section{MATERIEL ET METHODES}

Le' calcul des paramètres zootechniques et de la productivité du cheptel caprin du Delta a été effectué à partir des données recueillies au cours d'un suivi de troupeaux réalisé entre 1986 et 1990 dans 28 exploitations agricoles et sur un nombre d'environ de 1200 caprins, chaque animal étant identifié à l'aide d'une boucle auriculaire. Le corpus méthodologique utilisé, dénommé "Panurge", a été mis au point conjointement par le CIRAD-EMVT et l'ISRA $(3,5)$. Il se compose d'une base de données alimentée régulièrement par les informations collectées au cours d'un passage bimensuel dans chacune des exploitations agricoles suivies. Au cours de chaque passage, les différents événements démographiques survenus pendant la quinzaine précédente, tels que les mortalités, les naissances, les sorties et les entrées de caprins dans le troupeau, ont été relevés. Les pratiques d'allotement et de conduite, les performances de croissance des jeunes et la production laitière par chèvre et par troupeau ont également été enregistrées. C'est ainsi que pour chaque troupeau ont été notés les lieux successifs de pâturage, les types et niveaux de complémentation alimentaire, ainsi que les animaux bénéficiaires. Les jeunes ont été pesés au premier passage suivant la naissance, puis à chaque passage bi-hebdomadaire jusqu'à trois mois, puis mensuellement jusqu'à un an. Chacun des paramètres et indices présentés a été calculé indépendamment des autres, directement à partir des données de base à l'aide de programmes spécifiques élaborés par le CIRAD-EMVT.

La productivité des caprins du Delta a été comparée à celles des caprins de la Moyenne-Vallée du fleuve Sénégal (région de Kaedi en Mauritanie) et de la région de Louga située au sud du Delta pour les trois raisons suivantes : tout d'abord, les mêmes méthodes de collecte et d'analyse des informations à partir de "Panurge" ont été appliquées dans les trois régions. La comparaison des résultats obtenus a donc été aisée. Ensuite, les trois régions étaient dans la zone nord-sahélienne, c'est-à-dire dans un même contexte bioclimatique. Enfin, le matériel génétique était le même dans les trois régions et les pratiques d'élevage étaient assez semblables. Les effets des facteurs bioclimatiques pouvaient ainsi être considérés comme étant minimes sur les différences entre les trois régions. Il faut cependant mentionner une épidémie de fièvre de la Vallée du Rift, survenue dans le Delta en 1987, qui avait temporairement perturbé les cycles reproductifs des petits ruminants (10). Les productivités des troupeaux de la Moyenne-Vallée et de Louga ont respectivement été calculées à partir des données de François (4) et Moulin (7).

Dans cette étude, afin d'élargir la comparaison aux autres régions tropicales sèches, les paramètres des troupeaux caprins du Yatenga (Burkina Faso) et de Somalie établis par Bourzat (2) ont été pris comme références ainsi que ceux du Nordeste brésilien relevés par Quirin (9). Les productivités ont été également comparées avec celles calculées à partir des données de l'ISRA/CIRAD-EMVT (7, 8) pour les régions sénégalaises de Kaymor (Sine-Saloum) et de Kolda (Haute-Casamance), situées respectivement en zone soudanienne et en zone guinéenne.

Toutes les analyses statistiques ont été réalisées à l'aide du logiciel SPSS/PC+. Les paramètres de reproduction ont été analysés par la méthode des moindres carrés, les facteurs étudiés étant les suivants : année et saison de mise bas, rang, mode et année de naissance et existence d'une complémentation. Pour les paramètres de croissance, le facteur "sexe de l'animal" a été également pris en compte.

\section{RESULTATS}

Le tableau I présente les principaux paramètres zootechniques moyen du cheptel caprin du Delta, ainsi que les facteurs influant de manière significative sur le niveau de chaque paramètre. On peut constater l'effet significatif à fortement significatif des facteurs climatiques et biologiques les plus souvent cités dans les zones arides et semi-arides, tels que l'année, la saison, le rang de mise bas, le sexe et le mode de naissance. Il est intéressant de noter l'effet significatif du facteur technique que représente la complémentation, pratique d'élevage sur laquelle l'éleveur est en mesure d'agir pour modifier la productivité de son cheptel. Les troupeaux caprins complémentés présentaient de meilleurs paramètres de reproduction et de croissance que les autres troupeaux caprins du Delta. En outre, la mortalité y était moins forte, l'effet de la complémentation étant particulièrement sensible sur la mortalité avant le sevrage, c'est-à-dire entre la naissance et trois mois, notamment dans le cas de naissance gémellaire.

Le tableau II présente les paramètres zootechniques moyens qui ont permis de calculcr les productivités numériques et pondéralcs à trois mois des troupeaux caprins du Delta en fonction de la complémentation. Il présente également les paramètres et les productivités obtenues dans la Moyenne-Vallée par François (4) et dans la région de Louga par Moulin (7).

Les figures 2 et 3 comparent les profils moyens d'élaboration de la productivité numérique et pondérale moyenne selon le type de complémentation dans le Delta, ainsi que dans la Moyenne-Vallée et dans la région de Louga.

L'effet de la complémentation était très significatif sur le taux de fertilité (plus 19 points, soit 24 p. 100) et la mortalité avant-sevrage (moins 5 points, soit 41 p. 100), cela se traduisant par une meilleure productivité numérique en hausse de 26 points (plus de 35 p. 100). En revanche, la complémentation n'exerçait aucun effet décelable sur la prolificité, le poids à trois mois et le taux d'avortement. L'effet de la complémentation sur les autres paramètres (taux de mise bas et de fécondité, nombre de nés et productivité pondérale) découlait directement de l'amélioration induite sur le taux de fertilité et la mortalité avant-sevrage (0-3 mois).

Par ailleurs, les pratiques d'exploitations des caprins diffèrent entre les régions, au sein d'une même région entre les types d'éle- 
TABLEAU I

\section{Principaux paramètres zootechniques du cheptel caprin du Delta}

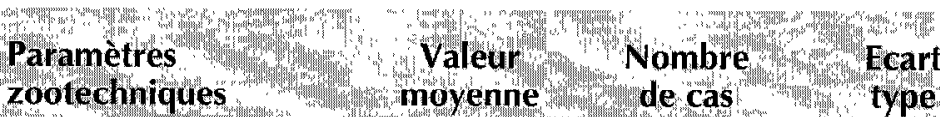

Facteurs de variation significatifs zootechniques

Age 1ère mise bas (j) de cas type

4 .

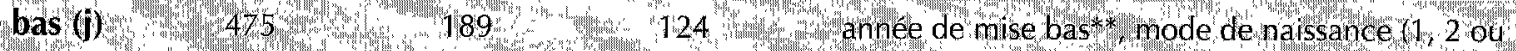
and

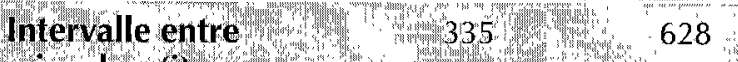

mises bas (0)

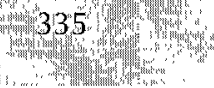

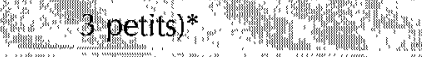

Taux de montalité win 190

(p. 100 du cheptel suivi)

Prolificite

Poids à 3 mois (kg)

Pint

Poids à 1 an (kg)
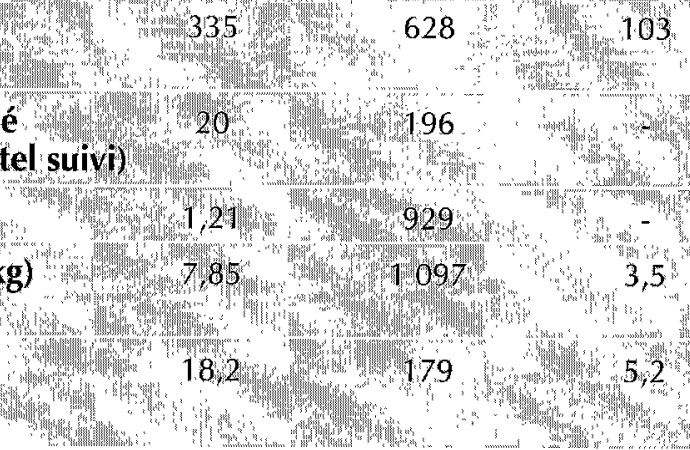

saison de la mise bas fil, annee etrang de la

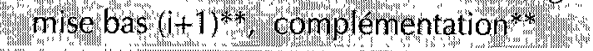
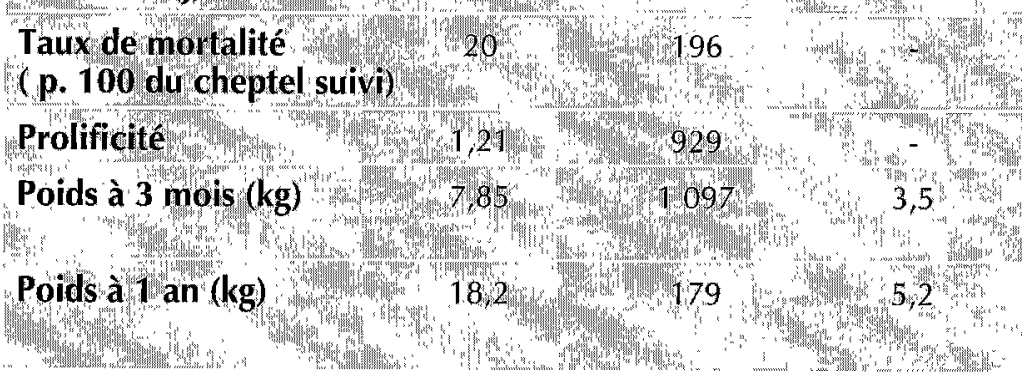

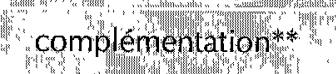
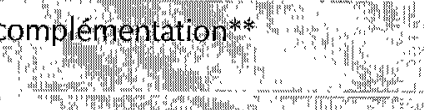

, n

sexex mode et saison de naissancex*

complementaton *t th

sexe**, saison de nạssance*

coinglenentation ${ }^{*}$

Seuil de signification : $\left({ }^{*}\right) \mathrm{P}=0,05$ et $($ **) $\mathrm{P}=0,01$

\section{TABLEAU II}

Les paramètres zootechniques et les productivités des caprins de la région Nord-Sénégal/Sud-Mauritanie

\section{complémentation}

taux de fertilite (\%)

taux davortement $(\%)$

taux de mises bas $(\%)$

prolificitét
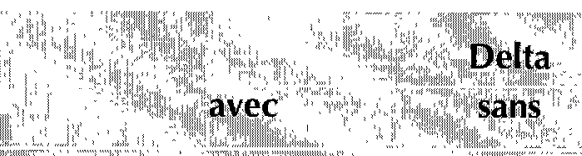

nombre de nés par an*

mortinataliter $(\%)$

(n)

in tau de féconditer (\%) mortalite $0-3$ mois $(\%) \quad 7$

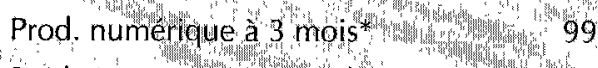

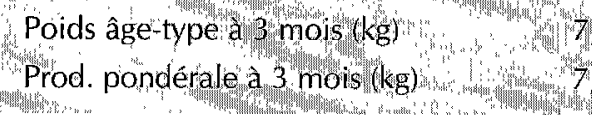

* pour 100 femelles mises à la reproduction

vage, et au sein d'un même type entre les mâles et les femelles. C'est ainsi que dans le Delta, les boucs sont commercialisés jeunes, autour de 4-5 mois. Le quotient d'exploilation des boucs est d'environ 75 p. 100 la première année, alors que, dans la même période, celui des chèvres n'excède pas 35 p. 100. (10). Il est donc délicat de comparer les productivités pondérales à un an qui reposent sur des effectifs mâles et femelles déséquilibrés.

Toutefois, dans les troupeaux complémentés, la productivité pondérale moyenne à un an, calculée sur les seuls caprins non exploités avant l'âge d'un an, était de $15,5 \mathrm{~kg}$, soit près du double de celle des troupeaux non complémentés, égale à $8,6 \mathrm{~kg}$. Cette différence en faveur des troupeaux complémentés reconnaît les mêmes origines que celles présentées pour la productivité à 3 mois, à savoir une meilleure fertilité et une moindre mortalité des jeunes.

\section{DISCUSSION}

Tout d'abord, il est intéressant de noter les faibles niveaux de production atteint dans ces systèmes. Même dans les cas de troupeaux complémentés, elle est en moyenne d'à peine un chevreau par an. Cette faible productivité, quoique normale pour la zone sahélienne, comme cela est montré plus loin, est liée à différents facteurs propres à la zone climatique. Parmi ceux-ci, il faut citer les facteurs alimentaires, en partie masqués par la technique de la complémentation, et génétiques.

L'effet très sensible de la complémentation des chèvres en lactation sur la mortalité avant-sevrage confirme l'intérêt de cette technique. Plus surprenants, son effet sur le taux de fertilité et 


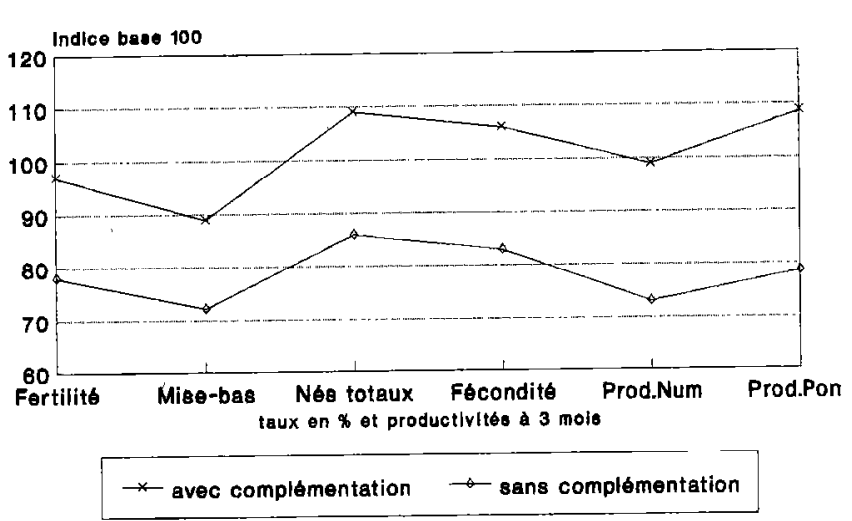

Indice taux - 100 ; indice mortalité - 0 ; indice pondéral - $7 \mathrm{~kg}$

Figure 2 : profil moyen d'élaboration de la productivité à trois mois des caprins du Delta : effet de la complémentation.

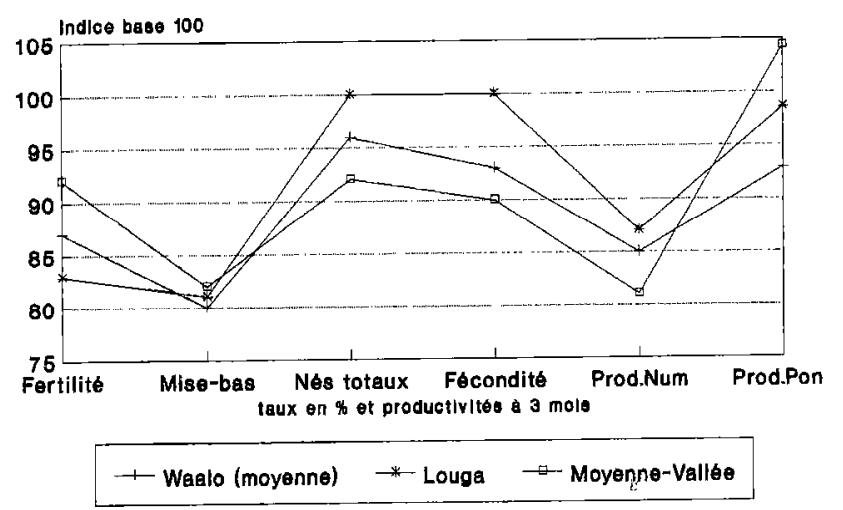

Indice taux - 100 ; indice mortalité - 0 ; indice pondéral - $7 \mathrm{~kg}$

Figure 3 : profil moyen d'élaboration de la productivité à trois mois des caprins par région.

l'absencc d'cffet sur la croissance méritent quelques explications supplémentaires.

La complémentation vise à améliorer l'état des chèvres en lactation et, en conséquence, à augmenter la production de lait. Dans le cas de traite, une partie du lait est prélevée, l'éleveur laissant au chevreau la quantité de lait nécessaire à sa croissance. Les chèvres ayant donné naissance à des jumeaux ne sont quasiment jamais traites, les produits bénéficiant ainsi de la totalité du lait de leur mère. La complémentation se traduirait donc par une diminution de la mortalité avant-sevrage en réduisant les mortalités d'origine nutritionnelle, en particulier dans le cas de naissance gémellaire. I 'ahsence d'effet de la complémentation sur le poids à trois mois des chevreaux suggère que les éleveurs accroissent leur prélèvement lorsque la production des chèvres augmente sous l'effet de la complémentation. La mise en place d'un contrôle laitier permettrait de confirmer ou d'infirmer cette hypothèse.

Le pic des mises bas se situe au début de la saison sèche, les trois mois de décembre, janvier et février comptant ensemble plus de 60 p. 100 des naissances (10). Ceci correspond à une période de fécondation en saison des pluies, durant les mois de juillet, août et septembre. En revanche, la lactation se déroule majoritairement au milieu et en fin de saison sèche, c'est-à-dire à l'époque où la contrainte alimentaire est la plus forte, tant d'un point de vue quantitatif que qualitatif. L'effet de la complémentation sur le taux de fertilité trouve probablement son explication dans la capacité des chèvres complémentées en lactation à maintenir un niveau suffisant de réserves corporelles et être ainsi fécondables au début de la période de lutte. L'effet très significatif de la complémentation sur l'intervalle entre mises bas (tableau I) semble confirmer cette hypothèse. Le contrôle de l'évolution de l'état corporel des animaux complémentés et non complémentés permettrait d'apporter une réponse définitive à cette question.

Les effets conjoints de la complémentation sur la fertilité et sur la mortalité avant-sevrage se traduisent respectivement par un gain de productivité numérique et pondérale d'environ 35 p. 100 et 38 p. 100 , ce qui est considérable.

En résumé, l'augmentation de la production laitière résultant de la complémentation diminue les mortalités des jeunes à l'allaitement, améliore la fertilité des chèvres en les maintenant en meilleur état durant la saison sèche et dégage probablement un surplus de lait prélevé dans le cas de traitc.

Le taux de fertilité moyen des troupeaux caprins du Delta (87 p. 100) se situe (tableau II) entre ceux relevés dans la Moyenne-Vallée (92 p. 100) et à Louga (83 p. 100). Cependant les troupeaux du Delta qui reçoivent un complément alimentaire, présentent le meilleur taux de fertilité (97 p. 100), alors que celui des troupeaux non complémentés s'avère le plus bas (78 p. 100).

La productivité numérique à trois mois des troupeaux complémentés est également supérieure aux productivités moyennes des troupeaux de la Moyenne-Vallée et de la région de Louga. En fait, dans ces deux régions, les écarts de productivité entre les systèmes d'élevage sont également importants, en relation avec le type de conduite retenu notamment dans le domáine de l'alimentation (7). Bourzal arrive à des conclusions semblables avec les troupeaux caprins du Yatenga (Burkina-Faso) et de Somalie.

Le poids moyen à trois mois relevé dans la Moyenne-Vallée $(9 \mathrm{~kg})$ est nettement supérieur à ceux constatés dans le Delta et la région de Louga, qui sont proches de $7,5 \mathrm{~kg}$. Contrairement aux résultats relevés dans les deux autres régions, les résultats pondéraux des caprins de la Moyenne-Vallée varient beaucoup en fonction des modes d'élevage (4). Dans les élevages citadins ou villageois comparables à certains élevages de la région de Louga, le poids moyen à trois mois est voisin de $8 \mathrm{~kg}$. Il est donc du même ordre de grandeur que ceux relevés dans le Delta et la région de Louga. En revanche dans les élevages pculs, lc poids moyen à trois mois est de $10,5 \mathrm{~kg}$, soit environ $3 \mathrm{~kg}$ de plus que celui des élevages peuls des deux autres régions. Or, dans les trois situations, il s'agit de la même race de caprins, et les modes de conduite des troupeaux sont assez semblables. Cette différence de poids doit être mise sur le compte de l'alimentation des chèvres allaitantes et des jeunes au sevrage, seul le type de parcours étant différent. En effet, pendant la période d'allaitcment, les caprins peuls de la Moyenne-Vallée pâturent les zones de culture de décrue après la récolte. Ils bénéficient ainsi d'une alimentation encore verte et donc riche, composée de restes de cultures et surtout de repousses de céréales (sorgho, maïs) et de légumineuses (niébé). Or, à la même époque, la majorité du cheptel caprin des régions de Louga et du Delta exploitent des parcours de faible valeur alimentaire.

L'ensemble des auteurs mentionne l'importance de la pathologie dans l'élevage des caprins en zone soudanienne et encore plus en zone guineenne. Cela se traduit par des taux d'avortement voisins de 30-35 p. 100, et des taux de mortalité avant-sevrage de l'ordre dc 12-14 p. 100. Toutefois, les troupeaux de ces zones climatiques 
présentent des productivités numérique et pondérale supérieures, de 50-100 p. 100, à celles des troupeaux des zones arides et semiarides, comme le montrent le tableau III et la figure 4.

En revanche, Quirin (9) dans le Nordeste brésilien trouve des paramètres de reproduction du même ordre de grandeur que ceux présentés par les troupeaux non complémentés du Waalo. Toutefois ces paramètres ont été calculés à partir de données collectées en période de sécheresse. Quirin (9), de même que Tourrand et coll. (11) estiment que les paramètres s'améliorent nettement lorsque les conditions climatiques s'approchent de la normale sans toutefois atteindre les performances des troupeaux des zones soudaniennes.

Ces diverses constatations viennent renforcer l'hypothèse d'un effet majeur de l'alimentation sur la productivité du cheptel caprin en zone tropicale sèche. Cependant, il semble qu'un haut niveau de complémentation permet à peine d'atteindre une productivité considérée comme moyenne en' zone climatique soudanienne ou guinéenne, tout en gardant à l'esprit qu'il s'agit de systèmes d'élevage relativement différents.

Il est clair que le fait de complémenter les caprins améliore de manière sensible leur productivité. Rapporté à l'échelle du troupeau, le gain numérique est d'environ 35 p. 100 et le gain pondéral d'environ 38 p. 100. Il s'agit de l'effet recherché par l'éleveur qui optimise ainsi le potentiel reproductif de son cheptel en anıéliorant les paramètres tels que la fertilité et la mortalité avant-sevrage tout en gardant un système technique traditionnel bien adapté aux conditions climatiques nord-sahéliennes, car basé sur le cycle saisonnier. En revanche, complémenter pour obtenir un désaisonnement des chèvres se traduisant par une époque plus favorable pour les chevreaux, n'est pas encore entré dans les mours. Il s'agit d'une pistc à explorcr unc fois résolue la contrainte de l'approvisionnement régulier en intrants nécessaires à la complémentation.

Cependant, la complémentation est un investissement. Ainsi, le prix moyen "rendu exploitation" de la farine basse de riz est-il d'environ 1 FF et le coût moyen de la complémentation d'environ $10 \mathrm{FF}$ par chèvre et par an, à raison de 200-300 $\mathrm{g}$ de farine basse de riz par jour et par chèvre, pendant 30 à 45 jours (10). Le prix
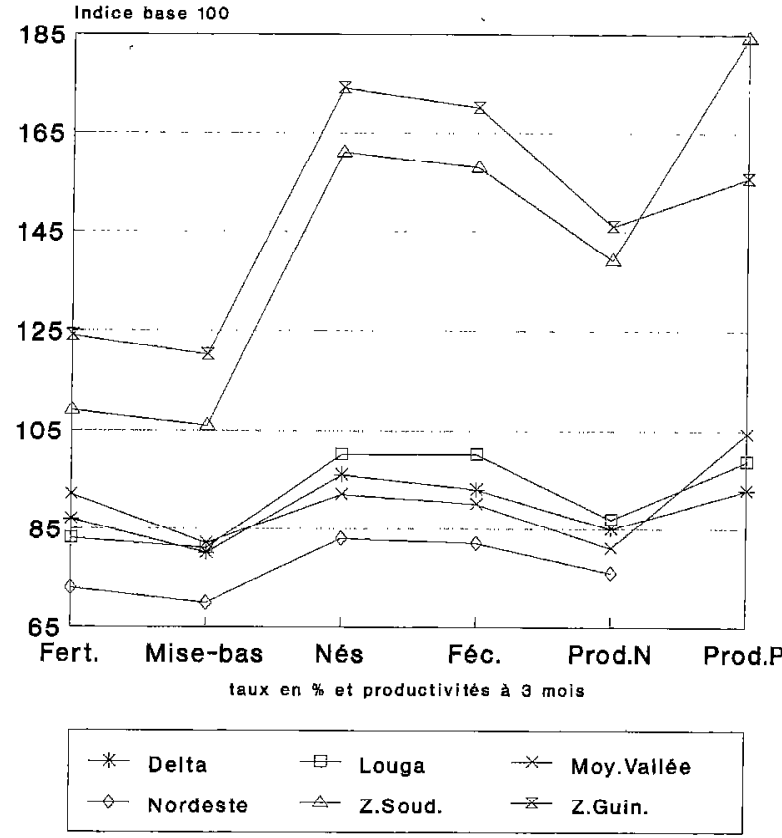

Indice taux - 100 ; indice mortalité -0 ; indice pondéral $-7 \mathrm{~kg}$

Figure 4 : profil moyen d'élaboration de la productivité à trois mois des caprins dans différentes zones climatiques.

moyen au départ de l'exploitation d'un chevreau de $10 \mathrm{~kg}$ et de 45 mois est de l'ordre de $90 \mathrm{FF}$. Le gain économique brut de la complémentation peut être évalué à environ 30-40 FF par chèvre et par an, ceci se traduisant à l'échelle du troupeau par un gain économique nel d'environ 20 p. 100, la rémunération de la maind'œuvre étant de l'ordre de 20 à $30 \mathrm{FF}$ par chèvre et par an. Dans les exploitations pratiquant la traite des caprins, ce revenu est augmenté de l'apport en lait auto-consommé ou vendu.

Le bénéfice de la complémentation doit ĉtrc comparé aux rémunérations des autres activités agricoles et salariées auxquelles peuvent prétendre les éleveurs. C'est ainsi que la pratique de la

\section{Les paramètres zootechniques et les productivités des caprins de diverses zones tropicales}

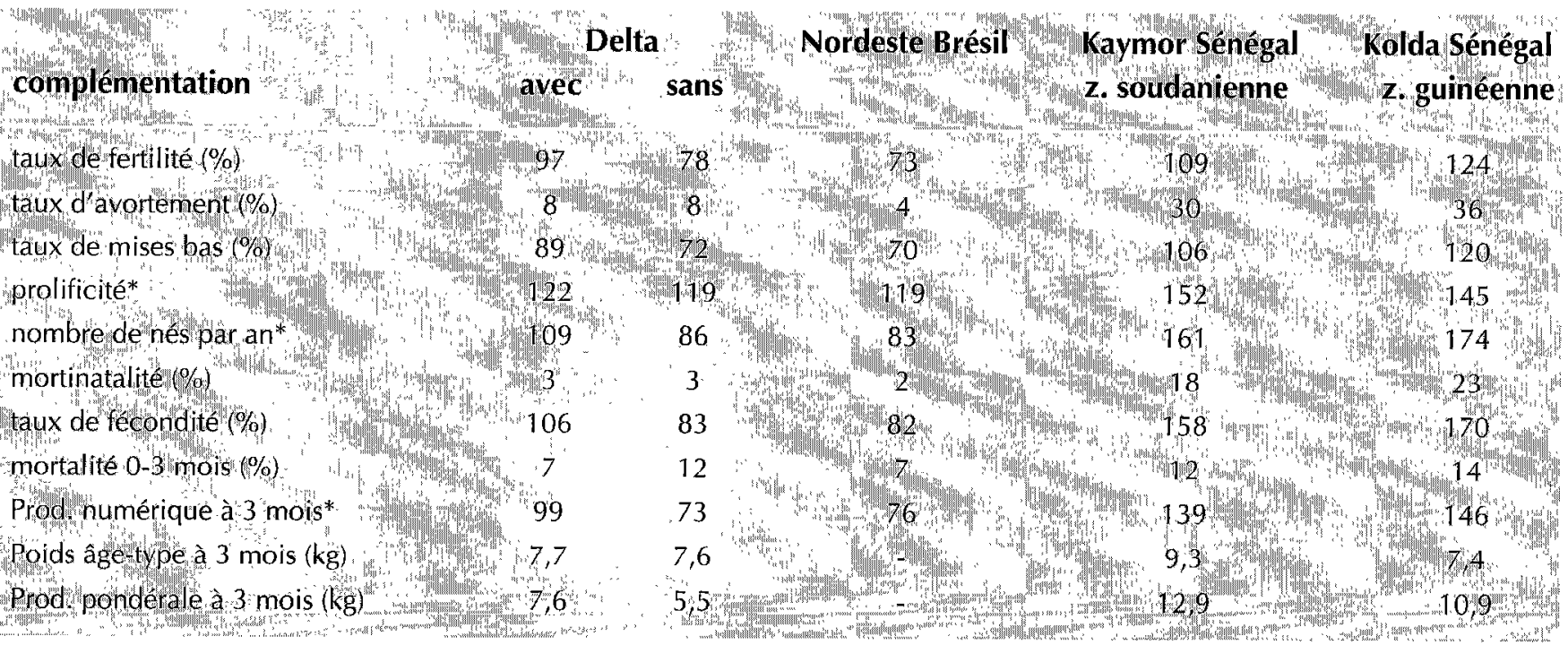


complémentation sur un troupeau de 100 chèvres, ce qui correspond à un troupeau d'environ 250 caprins, entraine une plus-value annuelle d'environ $1400 \mathrm{FF}$, soit près de 20 p. 100 d'un salaire annuel moyen* ou près de 30 p. 100 du revenu d'un hectare de riz irrigué bien conduit. Cette pratique est largement à la portée d'un adolescent qui peut difficilement exercer d'autres activités aussi rémunératrices. De plus, dans les exploitations agro-pastorales, la complémentation permet de valoriser les sous-produits agricoles issus des cultures irriguées, telles que les résidus d'usinage artisanal et les adventices de cultures (10).

Les éleveurs peuls proches des centres urbains pratiquent le plus la complémentation. A cela on peut avancer les deux principales raisons suivantes que sont la proximité d'un marché important et la facilité d'acquérir de la farine basse de riz ou des drêches de tomate, autre sous-produit agro-industriel fréquemment utilisé pour complémenter les petits ruminants. En effet, tout au long de l'année, ces éleveurs peuvent commercialiser les jeunes chevreaux sur le marché local, et acquérir ainsi les fonds nécessaires à l'achat d'intrants.

Par ailleurs, dans un contexte agricole en pleine mutation comme celui du Delta (6), cette meilleure valorisation de l'élevage caprin, à travers l'adoption de pratiques plus intensives, répond au souci partagé par de nombreux Peuls de diversifier leurs sources de revenus (10).

\section{CONCLUSION}

La pratique de la complémentation des chèvres dans le Delta du fleuve Sénégal entraîne un gain de productivité important, qui se traduit par un bénéfice économique conséquent, même dans les conditions particulières de cette région. A l'heure où l'on parle de systèmes agricoles durables, de réduction des coûts de production, de meilleure valorisation des ressources existantes et d'identité culturelle, le potentiel productif sous-exploité de l'élevage caprin, spécificité des pasteurs et agro-pasteurs peuls, peut ainsi constituer une voie de diversification intéressante.

\section{Summary}

Tourrand J.-F., Landais E. Goat productivity in farming production systems of the Senegal River Delta

Based on productivity analyses, the main zootechnical parameters of goat herds in the Senegal River Delta are presented here and compared with available data from the area and other tropical, ecological-regional areas. With an average annual production per goat under one kid $(0.85)$, wheighing a little less than $8 \mathrm{~kg}$ at three months, the results are relatively low, although in line with the area norms. Feeding habits, that enable Peul farmers of the Delta area to improve flock produclivily (by about 35-40\%) and thus the production protential value of their goat flock, are also described here.

Key words: Goat - Kid - Productivity - Feeding - Food enrichment - Animal husbandry - Zootechny - Senegal.

\footnotetext{
* La référence choisie étant le salaire annuel moyen d'un emploi dans une agro-industrie évalué à environ $8400 \mathrm{FF}$ (10)
}

\section{BIBLIOGRAPHIE}

1. AUDRU J., 1966. Etude des pâturages naturels et des problèmes pastoraux dans le Delta du fleuve Sénégal (tome 1 : description du milieu tome 2 : politique de l'élevage). Maisons-Alfort, France, IEMVT, 359 p.

2. BOURZAT D., 1991. Les petits ruminants dans les systèmes de production des zones aride et semi-aride de Somalie et du Burkina Faso. Maisons-Alfort, trance, ItMVI, 252 p. + annexes.

3. FAUGERE O., FAUGERE B., 1993. "Panurge". Suivi individuel dans les systèmes d'élevage traditionnel (Manuel). Maisons-Alfort, France, CIRAD-EMVT, ISRA-LNERV, 340 p. (Coll. Les logiciels en élevage)

4. FRANÇOIS J.L., 1990. Projet d'encadrement des éleveurs du Gorgol (Mauritanie) : résultats du suivi zootechnique des petits ruminants. Paris, France, Ministère français pour la coopération, $93 \mathrm{p}$.

5. LANDAIS E., FAUGERE O., 1990. Un modèle illustré de système d'investigation pour l'étude pluridisciplinaire des systèmes d'élevage en milieu traditionnel africain. Cah. Rech. Dév., $25: 75-94$.

6. LEGAL P.Y., 1992. Le Delta du fleuve Sénégal : une région en pleine mutation. Montpellier, France, CIRAD-SAR, 16 p. (n0-92)

7. MOULIN C.H., 1993. Performances animales et pratiques d'élevage en Afrique sahélienne. La diversité du fonctionnement des troupeaux de petits ruminants dans la communauté rurale de Ndiagne. Thèse doct., INA Paris-Grignon, Paris, France, 259 p.

8. MOULIN C.H., FAUGERE O., FAUGERE B., 1994. L'élevage traditionnel des petits ruminants au Sénégal. III. Pratiques de conduite et d'exploitation des animaux chez les éleveurs de la communauté rurale de Kaymor (Siné-Saloum, Sénégal). Revue Elev. Méd. vét. Pays trop., $47: 223-234$.

9. QUIRIN R., 1993. Epidemiologia e prevenção do aborto em caprinos. Petrolina-Pe, Brésil, EMBRAPA-CPATSA, 13 p.

10. TOURRAND J.F., 1993. L'élevage dans la révolution agricole au Waalo : ruptures et continuité. Thèse doc. Etat, Université Paris XII-Val de Marne, Créteil, France, $416 \mathrm{p}$.

11. TOURRAND J.F., CARON P., BONNAL, 1993. Recherche sur les systèmes d'élevage du semi-aride : cas du Nordeste brésilien. Montpellier, France, CIRAD-EMVT/SAR, $75 \mathrm{p}$.

12. TOURRAND J.F., LANDAIS E., 1994. Aménagements hydrauliques et développement : 'stratégies paysannes d'adaptation dans le Delta du fleuve Sénégal (1984-1991). Nat. Sci. Soc., 3:(2): 211-229.

Reçu le 19.1.96, accepté le 12.9.96

\section{Resumen}

Tourrand J.-F., Landais E. Productividad caprina en los sistemas de producción agrícola del Delta del Río Senegal

A través del análisis de la productividad, los autoreș presentan los principales parámetros zootécnicos del hato caprino del Delta del río Senegal, comparándolos con las referencias disponibles para la misma zona, así como algunas otras grandes zonas agro-ecológicas tropicales. Con una producción anual promedio por cabra inferior a un cabrito $(0,85)$, con un peso a tres meses de poco menos de $8 \mathrm{~kg}$, los rendimientos son relativamente bajos, aunque conformes a las normas regionales. Los autores presentan también las prácticas alimenticias que permiten a los productores peu; del Delta, mejorar sensiblemente la productividad de sus hatos, entre 35 y 40 por ciento y de valorar así el potencial productivo del hato caprino.

Palabras clave : Cabra - Cabrito - Productividad Alimentación - Enriquecimiento de los alimentos Ganadería - Zootecnia - Senegal. 